\title{
Animal models of chronic kidney disease: Screening tool to investigate nephroprotective effects of natural products
}

\author{
Sachinthi S. Amarasiri ${ }^{1}$, Anoja P. Attanayake ${ }^{2, *}$, Kamani A.P.W. Jayatilaka ${ }^{3}$, Lakmini K.B. Mudduwa ${ }^{4}$ \\ ${ }^{1}$ Lecturer, ${ }^{2}$ Senior Lecturer, ${ }^{3,4}$ Professor, ${ }^{1}$ Dept. of Medical Laboratory Science, ${ }^{2,3}$ Dept. of Biochemistry, ${ }^{4}$ Dept. of Pathology, \\ University of Ruhuna, Galle, Sri Lanka
}

*Corresponding Author:

Email: anoja715@yahoo.com

\begin{abstract}
Animals are used as experimental models to reproduce human diseases. To date, various animal models have been successfully developed by numerous methods to simulate human diseases including chronic kidney disease (CKD). Such models have played a central role in developing dialysis, transplantation experiments and more importantly in the discovery of new therapeutic agents from natural product for the care of patients with kidney disease. This review focuses on key information on in vivo models of CKD that have been developed through spontaneous, acquired and genetic approaches. Most of the experiments related to CKD have been carried out on rodent models such as mice and rats. Spontaneous disease models of CKD are generated by various metabolic and immunological methods. Nephrotoxic agents including adenine, adriamycin, cisplatin, folic acid, aristolochic acid and oxalate are used to induce CKD in addition to nephrectomy and unilateral ureteral obstruction models. Further, animal models developed through forward and reverse genetic approaches provide artificial models of CKD. Developing animal models to approximate human CKD is a challenging task since it requires reflecting the effect of age, sex, and comorbidities in addition to the disease condition. But, their usage to tease out the processes which can cause pathologic changes in a biological system is still important for the health care improvements related to CKD. However, no animal model can exactly simulate response in human CKD.
\end{abstract}

Keywords: Acquired methods, Animal models, Chronic kidney disease, Genetic approaches, Nephrotoxic agents, Spontaneous models.

\section{Introduction}

A model is an abstraction of a system, which is used to understand, explain or design aspects of interest. ${ }^{1}$ Scientific models are used to explain and predict a real phenomenon that is difficult to experience directly. ${ }^{2}$ Animal models are living experimental systems developed in one species for the purpose of studying phenomena occurring in another species. ${ }^{3}$ They have become an important research tool used for the better understanding of biology in human beings.

Most relevant information related to human anatomy and physiology could be derived from the study of human beings. According to Paul Beeson, progress in understanding and management of human disease must begin and end with studies of man. ${ }^{4}$ However, it is not always possible to obtain precise results in human studies related to biomedical sciences due to difficulties in controlling conditions and interfering variables. ${ }^{3,4}$ This issue could be minimized by using animals as models of humans. More scientific information could be generated in animal models than in clinical trials, which are conducted under controlled environmental conditions by changing one variable at a time. The health status of animals also could be easily monitored. Therefore, the practice of the modern science is to generalize findings of animal models to the real world situation by extrapolating the results to human beings. ${ }^{3}$

Scientists have used laboratory data derived from animal studies for closing knowledge gaps about health and disease in both humans and animals. Due to the bans regarding dissection of humans, physicians in ancient Greece dissected animals for anatomical studies. ${ }^{2}$ Most of the scientific knowledge regarding human physiology has been generated from the studies involving animals. Animal models were used in fundamental physiological discoveries such as demonstration of blood circulation, discovery of lung function, measurement of blood pressure etc. $^{2}$ They have played a central role in understanding disease processes and developing effective therapeutics such as antibiotics, insulin and vaccines. Most of the known hormones and their function in humans were discovered by animal experiments on endocrine glands. Studies on living animals were useful in understanding the vertebrate immune system which eventually facilitated organ transplantation in humans. ${ }^{5}$

Furthermore, animal experiments play a major role in the study of the disease pathogenesis of animals and humans. The principle behind the transmission of pathogens from one animal to another was first demonstrated by injecting blood from cows infected with anthrax virus to mice. Animal models of rheumatoid arthritis have been used to study the disease process and to test new treatments. Hepatitis $\mathrm{C}$ virus was discovered using a chimpanzee model. Chimpanzee models became useful in understanding the process behind transmission of HIV-1 virus in blood, blood products and body fluids. Animal models were used to study the disease process of poliomyelitis facilitating the development of polio vaccine. Moreover, animal experiments have contributed 
for many advances in cancer treatment. ${ }^{5}$

Use of animal models in understanding human anatomy and physiology has resulted in a paradigm shift in the fields of pharmacology, toxicology, immunology etc. There is a significant increase in the use of animals in fundamental as well as in diagnostic research in the $20^{\text {th }}$ century. $75-100$ million of vertebrates are used in research and testing purposes worldwide today. ${ }^{6}$

\section{Ethical and moral issues}

Animals experience pain and distress during experiments. Invasive procedures used in experiments, induction of diseases, lack of basic physiological needs, social deprivation and loss of the ability to fulfill natural behaviors may be harmful to the experimental animals. It has been proven that, animals show significant changes in physiological and hormonal markers of stress even in response to gentle handling. ${ }^{7}$ Therefore, scientists believe that, animal experiments should be carried out within an ethical framework.

Based on the above concept, Russell and Burch developed the principle of "Three Rs" - Replacement, Reduction and Refinement to assure wellbeing of the experimental animals. ${ }^{7}$ This approach provide an ethically and scientifically sound framework for the use of animals in biomedical sciences. ${ }^{2}$

Replacement refers to the use of non-animal alternatives to replace methods which use conscious living animals. ${ }^{2-7}$ In vitro techniques such as cell culture and tissue culture, use of computerized models may fulfill the above requirement. ${ }^{6}$ However, it is difficult to replace animal models completely to achieve same scientific aim. Therefore, scientists focus more about reduction and refinement as humane experimental techniques. Gathering scientific data using a minimum number of animals required refers to "Reduction". But it is also important to use a sufficient number of animals to generate statistically precise and significant results to prevent repetition of experiments. ${ }^{8}$ Refinement refers to the procedures that minimize potential pain, suffering or distress to the experimental animals. This could be achieved by improving animal husbandry and housing, careful handling of the animals, and administration of appropriate anesthetics and analgesics during the experiments etc. ${ }^{2-8}$ Furthermore, proper education and training of the researchers and the technical personnel involve in animal experiments may improve the wellbeing of experimental animals. ${ }^{6}$

\section{Animals used as human models}

Different types of animals have been used to reproduce human diseases. Selection of a suitable animal model is a complex as well as an expensive process. An ideal animal model should possess similar anatomical and physiological features to humans. In addition, the pathological findings of human disease should be evident in animal models and the findings of animal experiments could be precisely transferable to humans. ${ }^{9}$
Considering the above factors, monkeys and chimpanzees are the ideal models of humans due to genetic, structural and functional similarities. However, the use of above species in animal research is minimized as a consequence of ethical issues. Similarly, ethical considerations have limited the use of dogs in research purposes despite the fact that they have been successful in developing dialysis and transplantation experiments. ${ }^{10}$

The similarities in the cell structure and function in all vertebrates help researchers to design experiments using non-mammalian vertebrates (frogs, fish and chickens) and mammalian vertebrates (mice, rats, rabbits, cats, dogs and primates). ${ }^{5}$ Scientists hypothesize that mice, rats, and humans inherited genes from a common mammalian ancestor. The genetic similarities between rodents and humans, their short reproduction period, easy and cheap availability of them and flexibility of genetic manipulation make rodents ideal as the models for studying human disorders. ${ }^{9,11,12}$ Therefore, approximately $80 \%$ of the animal experiments carried out on vertebrates involved mice or rats. 5,6

Mouse model is the most widely used animal model in laboratory research. Possibility of genetic modification and the use of sophisticated transgenic approaches for accurate recapitulation of the pathology of human diseases are the additional advantages in mouse models. These approaches are widely used in developing cancer models. ${ }^{13}$ However, the use of mice is limited in laboratory surgical intervention experiments due to their smaller size. Rat models share similar advantages to mice. They are inexpensive compared to other large animal models like rabbits, cats, dogs, pigs etc. Further, the short gestation period of 59-72 days permits production of large number of animals within a short period of time making them suitable as experimental models. ${ }^{9}$

The variance in animal experiments could be minimized by using a single species of a rodent model, of the same gender at a specific age. ${ }^{10}$ Through the process called "inbreeding" genetically identical animals could be made available for experimental use. ${ }^{14}$ Inbred strains are more stable, uniform, repeatable and better defined. ${ }^{10}$ However, selection of the most informative model is a challenge due to the inherent differences between strains. Thus, strain selection is a major consideration in animal modeling, especially in rodents. ${ }^{14}$

\section{Animal models of chronic kidney disease (CKD)}

Chronic kidney disease (CKD) remains one of the serious global health problems. It is a slowly progressive disorder that might lead to end-stage renal disease (ESRD). ${ }^{15}$ There is an increasing prevalence of patients with kidney disease worldwide with adverse outcomes such as kidney failure, cardiovascular disease, and premature death. ${ }^{16}$

Due to paucity of effective therapies targeting 
kidney functions and side effects of existing pharmaceutical agents, novel approaches to develop new therapeutic strategies have become a health care priority. ${ }^{15}$ Since the pathological process underlying CKD affects diverse systems of the body, CKD patients may present with complications involving multiple organs and functions. ${ }^{17}$ Thus, it is difficult to study the disease using human subjects. Therefore, in vivo animal models play a significant role as an experimental tool in examining the biochemical, physiological and histopathological mechanisms of CKD. ${ }^{15}$ Among them, rodent models remain as the most popular species to approximate human disease..$^{10}$

The primary measure of kidney function is glomerular filtration rate which is derived from serum and urine creatinine concentrations. Reduction of glomerular filtration rate (GFR) by 50\% defines CKD in animal models. However, GFR cannot be reliably estimated in animal models. Therefore, in animal studies of $\mathrm{CKD}$, significant changes in biomarkers of renal function including blood urea nitrogen (BUN) and creatinine are used to assess the kidney function. In addition, urinary protein excretion, presence of typical complications associated with CKD in humans such as normochromic anemia, hyperphosphatemia, hyperparathyroidism, hyperkalemia etc. are the features assessed in animal models of CKD. ${ }^{18,19}$ Furthermore, the findings related to histopathology includes; development of glomerulosclerosis, tubulointerstitial inflammation and fibrosis. ${ }^{12}$

A reliable model which produces features of stable disease within a relatively short period of time is required for the interventions related to CKD. A method that work both on male and female animals with low inter individual variability would be ideal in developing animal models to simulate human CKD. ${ }^{19}$ Various animal models have been developed using numerous methods in the area of CKD to approximate the human disease. This review focus on key information on in vivo models of CKD developed through spontaneous, acquired and genetic methods.

\section{Spontaneous models}

Spontaneous disease models could be generated by various metabolic or immunological methods.

1. Lupus nephritis: Development of Lupus nephritis by immune complex-mediated glomerulonephritis is one such example. Mouse models including MRL/lpr and NZB/W have the capacity to develop lupus nephritis spontaneously resembling human histological findings related to CKD. ${ }^{20}$ Studies in mouse models of lupus nephritis have been useful in understanding the primary pathogenesis of autoimmune kidney disease. Death due to renal failure has been identified as an important end-point of these studies. ${ }^{21}$

2. Aging: Since aging can cause progression of renal impairment and scarring, rats older than 24 months age could be used as a model of CKD. It is preferred to use male rats of this model due to early and severe symptoms of scarring than in females..$^{20}$

3. Spontaneously hypertensive rats: Spontaneously hypertensive rats are another experimental model that can be used in research purposes related to CKD. Biochemical and histopathological features related to CKD could be observed starting from $6^{\text {th }}$ week in the spontaneously hypertensive rat model. ${ }^{20}$

4. CKD as a consequence of acute kidney injury: The relative risk of CKD after a severe episode of ischemic acute kidney injury is useful in developing animal models of CKD. Long-term interstitial fibrosis and progressive renal insufficiency are the characteristic features of this model. This model is investigated most commonly in rats and mice. Renal fibrosis is evident during the chronic phase of the disease. ${ }^{21}$

5. Congenital deficiency of nephrons: Munich Wistar Frömter rat is a genetic model with congenital deficiency in nephron number. Their nephron number is $30-50 \%$ less than normal. These animals are predisposed to develop hypertension in adulthood. Munich Wistar Frömter rats develop proteinuria at ten weeks of age and exhibits significant glomerulosclerosis by nine months. ${ }^{20}$

\section{Acquired models}

Animal models of CKD has been developed using surgical as well as chemical methods causing nephrotoxicity.

\section{Surgical methods}

1. 5/6 Nephrectomy: Reduction in renal mass experimentally by subtotal nephrectomy cause progressive glomerulosclerosis and tubulointerstitial fibrosis associated with CKD. It is an easy method of inducing disease in larger animals such as rats and rabbits. Several methods are available to develop CKD using this model. One approach is the uninephrectomy followed by ligation of polar branches of the renal artery. It is a method commonly performed in rats. Another approach is the surgical excision of approximately $50 \%$ of the remaining kidney, 1-2 weeks after uninephrectomy. This approach can be used both in rats and mice. A combination of the above two models, which ties one or more branches of the mouse renal artery, and then remove additional renal mass to achieve a total 5/6 nephrectomy is also an available approach for developing CKD in animals. ${ }^{17-20-22}$

The nephrectomy method is highly dependent on surgical expertise and the availability of operating facilities. ${ }^{23}$ Since the progression of renal failure is closely related to the amount of tissue infarcted or excised, it is associated with relatively large inter-individual and inter-laboratory variations. ${ }^{20-23}$ Further, the C57BL/6 strain mice, 
which is commonly used in animal experiments, seems to be relatively fibrosis resistant following $5 / 6$ nephrectomy. The strains showing susceptibility include; CD-1, 129Sv, and Swiss-Webster. ${ }^{21}$ Reductions in tissue mass available for analysis, substantial risk of mortality and phenotypic alterations associated with procedures have compromised the use of this method in development of CKD in animal models. ${ }^{24}$

2. Unilateral ureteral obstruction (UUO): This is an in vivo model useful in examining the mechanisms of tubulointerstitial fibrosis associated with human renal disease. ${ }^{20}$ It is possible to induce this model both in rats and mice without specific strain dependence. ${ }^{25}$ Reduction in renal blood flow and glomerular filtration rate is remarked within $24 \mathrm{hrs}$ following kidney obstruction. Interstitial inflammation peaks at 2-3 days and tubular dilation, tubular atrophy and fibrosis is initiated from one week after UUO. The experimental model would reach the end stage by around two weeks following the procedure. ${ }^{20-23}$

The UUO model of CKD has several advantages over nephrectomy model. The availability of the contralateral kidney as a control, lack of a "uremic" environment, variation in the severity, timing, and duration of obstruction, as well as the opportunity to study recovery following relief of the obstruction are few of them. ${ }^{26}$ There are few limitations as well. The requirement of a survival surgery, compensation by the normal contralateral kidney which prevent meaningful renal functional measures and the absence of urine from the damaged kidney for investigation of biomarkers are few of them. Increase of renal vascular resistance after the surgery result in reduction of renal blood flow leading to differences in renal delivery rates of exogenous therapeutic agents is another limitation that should be considered during experiments related to therapeutic agents in this model. ${ }^{21}$

Reversible unilateral ureteral obstruction is a modification of the above model with several additional advantages. The principle behind developing this model is to minimize injury to the ureter, allowing return of patency after relief of complete obstruction. It is a method that uses simple surgical techniques that can be performed by laboratory personnel with basic technical skills. Assessment of renal function is possible using biochemical analysis of serum and urine samples during kidney injury as well as during repair. Reversible unilateral ureteral obstruction permits translational and long-term studies in living animals with CKD as well. ${ }^{25}$

\section{Animal models of chronic nephrotoxicity}

Considering the limitations in surgical models, the use of non-surgical options that use nephrotoxic agents seems beneficial. Following are few examples for the animal models developed using nephrotoxic agents in recent publications.

1. Adenine induced CKD: Development of an animal model for CKD based on the intake of adenine was first proposed by Yokowaza in 1982..4 Oral administration of adenine mixed with the food at a concentration of $0.75 \%, \mathrm{w} / \mathrm{w}$ was performed for the development of the experimental model. Both adenine and its metabolite, 2,8-dihydroxyadenine, precipitate in the renal tubules and form crystals causing degenerative changes in renal tubules and the interstitium. The consumption of oral adenine causes occlusion of renal tubules which retards excretion of nitrogenous substances. This leads to morphological, biochemical and histopathological alterations in kidneys resembling CKD in humans. ${ }^{23-27}$ Adult animals develop hyperphosphatemia, secondary hyperparathyroidism, bone disease, and vascular calcification following oral administration of adenine. ${ }^{15-24}$

High morbidity and mortality was observed in above cases due to starvation and malnutrition rather than renal failure in animals. As a solution, Ting et $a l{ }^{23}$ mixed adenine in a casein-based chow, in which the casein effectively removed the inherent smell and taste of adenine.

Another new model was developed by Mohammed et al for the induction of CKD via intraperitoneal adenine administration to overcome the high morbidity and mortality. ${ }^{13}$ It seems to be a superior model to oral adenine administration for the induction of CKD since, it is more practical, convenient and accurate due to direct entry of the drug to the systemic circulation. ${ }^{15}$

Adenine model is a better alternative for the surgical models of CKD due to several reasons. It is a convenient method which can be used to induce renal failure, with very little inter-individual variation over a short period of time..$^{27}$ The degree of reduction in renal function is also relatively homogeneous. ${ }^{24}$

2. Adriamycin induced CKD: Adriamycin (doxorubicin) is an anthracycline drug used in cancer chemotherapy. It is a cytotoxic antibiotic isolated from cultures of Streptomyces peucetius var. caesius. It is a well-known inducer of renal injury in rodents, which mimic the features in human CKD. Adriamycin is not significantly metabolized and accumulated mainly in the kidney causing nephrotoxicity. The optimal dose of adriamycin which may indicate nephrotoxicity depends on species, strain, gender, age, source and batch of the experimental animals. The preferable route of administration in most of the studies is intravenous using the tail vein. However, skin necrosis in the event of tissue extravazation is a 
main complication associated with tail vein injection. Substernal intracardiac approach, intrarenal route, direct injection of the renal artery and intraperitoneal administration are the other routes of administration. Even though intraperitoneal administration is an easy method to deal with the drug, due to variable absorption through the peritoneal membrane and inconsistency in induction of renal injury compared with the intravenous route makes this method less favorable. Male rats are more susceptible than female rats to adriamycininduced nephropathy. ${ }^{12}$

Adriamycin-induced nephropathy is associated with the histopathological changes related to human CKD. ${ }^{28}$ The changes appear as early as one to two weeks after adriamycin injection, and get severe by four weeks. The experimental rodents present with elevated serum concentration of creatinine, reduced creatinine clearance, reduced concentration of serum albumin and increased urine protein excretion following administration of the drug. ${ }^{12-29}$ Although features of acute tubular necrosis could be observed with many nephrotoxic drugs, adriamycin result in chronic effects on the kidney as shown by Okuda et al. ${ }^{28}$ in their study.

Adriamycin induced nephropathy model is a highly reproducible model of renal injury with acceptable mortality and morbidity. Even the timing of renal injury is consistent and predictable in this model. However, the narrow therapeutic index of adriamycin leads to a large variation in disease severity even by a small difference in the dose administered. A $0.5 \mathrm{mg} / \mathrm{kg}$ difference than the optimum dose may lead to either lack of renal injury or mortality in the experimental animals. Although the model is consistent and reproducible, still there is possibility for some individual variability in response, even within the same strain of rodents. ${ }^{12}$

3. Diabetic nephropathy: Diabetic nephropathy is a major cause of human CKD. Several mouse models have been developed to approximate the pathogenesis of diabetic nephropathy. The pancreatic $\beta$ - cell toxin streptozotocin (STZ) is commonly used to model Type 1 diabetes in order to develop nephropathy in experimental animals. Structural similarity between glucose and STZ results in transportation of STZ into pancreatic $\beta$ cells, with subsequent destruction leading to hyperglycemia. Mice are injected with a higher dose of STZ on consecutive days due to their resistance to STZ than in rats. Further, Akita mice are used to develop type 1 diabetes mellitus. A spontaneous point mutation in the Akita mice leads to misfolding of insulin, resulting in pancreatic $\beta$-cell failure. This may result in sustained hyperglycemia with high levels of albuminuria and nephropathy related renal histopathological changes. ${ }^{11}$ In addition to above mouse models, CD1 and eNOS-/- C57BL/6 mice also have been reported to develop severe nephropathy after STZ administration. However, it may take several months to develop renal fibrosis after STZ treatment. ${ }^{21}$

The most common type of diabetes which may lead to nephropathy is type 2 diabetes. Animal models of type 2 diabetes have been developed mainly from single-gene mutations. ${ }^{11}$ The $\mathrm{db} / \mathrm{db}$ mouse model is one such example which resulted from mutations of leptin receptor. This model develops nephropathy with focal interstitial fibrosis with age. ${ }^{21}$ Similarly, Zucker rats which are characterized by mild glucose intolerance and peripheral insulin resistance are used to reproduce type 2 diabetes leading to albuminuria and glomerular injury. ${ }^{11}$

4. Other models of chronic nephrotoxicity: Environmental toxins, heavy metals and alternative medicines are reported as potential causes of CKD in humans. Based on above findings, several animal studies have been conducted to induce nephrotoxic renal disease.

Folic acid and aristolochic acid nephropathy are two such examples which cause acute kidney injury that may progress to interstitial fibrosis. ${ }^{21}$ Intraperitoneal administration of folic acid in a high dosage $(250 \mathrm{ug} / \mathrm{g} \mathrm{BW})$ is a common method of inducing patchy interstitial fibrosis in the chronic phase (28-42 days). ${ }^{21-30}$ Intraperitoneal injection of aristolochic acid weekly has led to progressive fibrosis and kidney failure severely in male mice in the studies involving this model. The results were similar to the findings in adenine induced models. ${ }^{19}$

Long term usage of cyclosporine A can induce renal fibrosis in human. Based on this theory, a rat model has been developed by administering cyclosporin A $(7.5 \mathrm{mg} / \mathrm{kg} /$ day and $15 \mathrm{mg} / \mathrm{kg} / \mathrm{day}$ s.c.) for 28 day period in the studies involving CKD. ${ }^{301}$

Cisplatin

(cDDP;

cisdiamminedichloroplatinum II) is another potent anticancer agent used in the treatment of solid tumors. Renal impairment is the most serious adverse effect reported in patients treated with cisplatin. These findings have led to the development of cisplatin induced nephrotoxic models for the experiments on CKD. ${ }^{31}$

The oxalate diet induced model is another reliable option. Unlike in oral adenine administration, oxalate is readily eaten by mice. Further, measurement of urinary oxalate excretion is useful in precise monitoring of dietary intake. Short time frame (one to three weeks) for the induction of advanced CKD is an additional advantage of this model. ${ }^{19}$

5. Radiation nephropathy: Animal models with nephropathy could be generated by therapeutic irradiation. However, the possibility of inducing 
disease is less $(20 \%)$ compared to the other methods of induction. Acute endothelial injuries, chronic progressive secondary sclerosis with proportional tubulointerstitial fibrosis are the characteristic features of radiation nephropathy. ${ }^{20}$

\section{Genetically engineered models}

Mouse models of autosomal dominant and autosomal recessive kidney disease which develop CKD are available. One such example is the genetic deletion of the gene encoding the $\alpha 3$ chain of collagen IV in mice in which results in an autosomal recessive form of CKD. ${ }^{21}$

Forward genetic approaches including transgenic and knockout studies have been successfully used in rat models for the investigation of biological function of genes in CKD. ${ }^{18}$ Rat is considered a good model system for the genetic analysis of CKD using reverse genetic approaches like quantitative trait loci (QTL) analysis. QTL analysis is useful to identify primary genetic determinants of kidney disease. Genetic studies in rats have already identified many QTL. The repeated findings of QTL in the same region in different crosses suggest involvement of several key loci in kidney disease. Rat models have become ideal candidates for genetic analysis due to less genetic complexity, better genetic tools, and a controlled environment. ${ }^{10-18}$

The genetically engineered animal models proven to be useful in CKD studies include; integrin-linked kinase and phosphoinositide3-kinase C2alpha knockout mice, podocin, $\alpha$-actinin-4, dicer, VEGF-A, semiphorin A, NFAT, sidekick-1, Notch-1, angiopoietin-2 mice etc. These models are reported with abnormal podocyte function, podocyte-depletion and chronic renal insufficiency. ${ }^{21}$

Several single HIV gene transgenic and gene deletion models have been generated to determine the role of specific HIV genes in the pathogenesis of HIVassociated nephropathy (HIVAN). The first HIVAN transgenic mouse model is the transgenic 26 (Tg26) mice. These models develop proteinuria at 24 days of age. Considerable mortality rate $(20 \%)$ was observed in these mice between two to six months and histopathological features of focal changes were noticed in mice younger than two months of age. ${ }^{20}$

Unlike in the animal models discussed previously, transgenic animal models provide an artificial model of CKD. Their use is limited due to high cost and limited accessibility to these animals. ${ }^{27}$

\section{Discussion}

Animal models accounts for the most of the recent improvements related to the care of patients with kidney disease. Sheep models have been beneficial in the development of the arteriovenous shunt useful in the hemodialysis treatment of CKD patients. Animal experiments on mice, guinea pigs and dogs were useful in understanding the immune basis of graft rejection during kidney transplantation as well as for the development of effective drugs to suppress rejection reactions. Discovery of the immunosuppressive properties of the new drug cyclosporine is one such effective treatment method which is useful during kidney transplantation in animal models. Furthermore, studies on dog models were worthwhile in developing the novel treatment method of lithotripsy, where the kidney stones are removed without a surgery. ${ }^{32}$

Even though scientific information could be generated, an animal model can never be the perfect match for a human disease. Although, hundreds of experiments are carried out on developing effective therapeutics for kidney patients using animal models, most of them fail in clinical studies. Due to the differences in species, low incidence of subjects and artificial induction of the disease, misleading results could be produced in animal studies. ${ }^{34}$ Further, the nondetectable effects in animals such as minor headaches or hallucinations may remain as a limitation of animal studies. $^{6}$

No animal model can exactly simulate or predict response in human CKD. It is insufficient to reflect only the disease condition in chronic diseases and effect of age, sex, and comorbidities should also be considered during developing animal models. ${ }^{35}$ Therefore, developing animal models to approximate human CKD is a challenging task. However, history has proven that, the experiments on large animal models have been exceptionally successful as predictive models for humans. ${ }^{36}$ Even though animal experiments are incapable of reproducing same pathological consequences of human disease, their usage to tease out the processes which can cause pathologic changes in a biological system should be appreciated.

\section{References}

1. Dori D. "Object-process methodology: A holistic system paradigm". New York. 2002. [Springer].

2. Franco HN. "Animal experiments in biomedical research: A historical perspective" Animals 2013;3:238-73.

3. Van der Staay FJ. "Animal models of behavioral dysfunctions: Basic concepts and classifications and an evaluation strategy" Brain Res Reviews 2006;52:131-59.

4. Anderegg C, Archibald K, Bailey J, Cohen MJ et al. "A critical look at animal experimentation" Medl Res Modernization Comittee 2006;19:1-34.

5. Nuffield Council on Bioethics. "The Ethics of Research Involving Animals" 2005.

6. Baumans V. "Use of animals in experimental research : an ethical dilemma?" Gene Therapy 2004;11:964-6.

7. Ferdowsian HR, Beck N. "Ethical and scientific considerations regarding animal testing and research" Plos One 2011;6:1-4.

8. Festing S, Wilkinson R. "Talking point on the use of animals in scientific research" EMBO Reports 2007;8:526-30.

9. Grossman RC. "Experimental models of renal disease and the cardiovascular system" Open Cardiovascular Med J 2010;4:257-64.

10. Becker GJ, Hewitson TD. "Animal models of chronic kidney disease: Useful but not perfect" Nephrology 
Dialysis Transplantation 2013;28:2432-38.

11. Breyer MD. "Mouse models of diabetic nephropathy" $J$ Am Soc Nephrol 2004;16:27-45.

12. Lee VW, Harris DC. "Adriamycin nephropathy: A model of focal segmental glomerulosclerosis" Nephrol $2011 ; 16: 30-8$.

13. Lieschke GJ, Currie PD. “Animal models of human disease : zebrafish swim into view" Nature Reviews Genetics 2010;8:353-67.

14. Ericsson AC, Crim MJ, Franklin CL. "A brief history of animal modeling" Missouri Med 2013;110:201-05.

15. Abi M Al, Busaidi M Al, Yasin J, Schupp N, Nemmar A, Ali BH. "Development of a new model for the induction of chronic kidney disease via intraperitoneal adenine administration, and the effect of treatment with gum acacia thereon" Am J Translational Res 2015;7:28-38.

16. Sabljar M. "Pathophysiology and classification of kidney diseases" $J$ Int Federation Clin Chem 2009;20:2-11.

17. Barbosa E, Schettert I, Ii S, Loprete JA, Schiaveto A et al. "Animal model of chronic kidney disease using a unilateral technique of renal ischemia and reperfusion in White New Zealand rabbits" Acta Cirúrgica Brasileira 2014;29:651-57.

18. Korstanje R, DiPetrillo K. "Unraveling the genetics of chronic kidney disease using animal models" Am J Physiol- Renal Physiol 2004;287:347-52.

19. Mulay SR, Eberhard JN, Pfann V, Marschner JA, Darisipudi MN, Daniel C et al. "Oxalate-induced chronic kidney disease with its uremic and cardiovascular complications in C57BL / 6 mice" Am J Physiol-Renal Physiol 2016;310:785-95.

20. Yang HC, Zuo Y, Fogo AB. "Models of chronic kidney disease" Drug Discovery Today: Dis Models 2010;7:139.

21. Eddy AA, Okamura DM, Yamaguchi I, López-Guisa JM. "Investigating mechanisms of chronic kidney disease in mouse models" Pediatric Nephrol 2012;27:1233-47.

22. Shimamura T, Morrison AB. "A progressive glomeruloscierosis occurring in partial five-sixths nephrectomized rats" American Journal of Pathology (1975) 79, 95-106

23. Jia T, Olauson H, Lindberg K, Amin R, Edvardsson K et al. "A novel model of adenine-induced tubulointerstitial nephropathy in mice" BMC Nephrol 2013;14:1-8.

24. Claramunt D, Gil-peña H, Fuente R, García-lópez E, Loredo $\mathrm{V}$ et al. "Chronic kidney disease induced by adenine: a suitable model of growth retardation in uremia" Am J Physiol- Renal Physiol 2015;309:57-62.

25. Puri TS, Shakaib MI, Chang A, Mathew L, Olayinka O et al. "Chronic kidney disease induced in mice by reversible unilateral ureteral obstruction is dependent on genetic background" Am J Physiol-Renal Physiol 2010;298:1024-32.

26. Chevalier RL, Forbes MS, Thornhill BA. "Ureteral obstruction as a model of renal interstitial fibrosis and obstructive nephropathy" Kidney Int 2009;75:1145-52

27. Tani T, Orimo H, Shimizu A, Tsuruoka S. "Development of a novel chronic kidney disease mouse model to evaluate the progression of hyperphosphatemia and associated mineral bone disease" Scientific Reports 2017:1-12.

28. Okuda S, Oh Y, Tsuruda H, Onoyama K, Fujimi S, Fujishima M. "Adriamycin-induced nephropathy as a model of chronic progressive glomerular disease". Kidney Int 1986;29:502-10.

29. Wang Y, Wang YP, Tay YC, Harris DCH. "Progressive adriamycin nephropathy in mice: Sequence of histologic and immunohistochemical events" Kidney Int
2000;58:1797-804.

30. Yamate J, Ishida A, Tsujino K, Tatsumi M, Nakatsuji S et al. "Immunohistochemical study of rat renal interstitial fibrosis induced by repeated injection of cisplatin, with special reference to the kinetics of macrophages and myofibroblasts" Toxicol Pathol 1996;24,199-206.

31. Behling EB, Sendão MC, Francescato HDC, Antunes MG, Costa RS, Bianchi MDLP. "Comparative study of multiple dosage of quercetin against cisplatin-induced nephrotoxicity and oxidative stress in rat kidneys" Pharmacol Reports 2006;58:526-32.

32. Couser WMD. "Animal research essential in fight against kidney disease".

33. Furness PN, Harris K. "An evaluation of experimental models of glomerulonephritis" Int J Exp Pathol 1994;75,9-22.

34. Van Der WHB, Howells DW, Sena ES, Porritt MJ, Rewell S et al. "Can animal models of disease reliably inform human studies?” PLos Med 2010;7:1-8.

35. Shanks N, Greek R, Greek J. "Are animal models predictive for humans?" Philosophy Ethics Humanities Med 2009;20:1-20. 